\title{
Low-dielectric, nanoporous polyimide thin films prepared from block copolymer templating
}

\author{
C. Wang, T. M. Wang, Q. H. Wang* \\ State Key Laboratory of Solid Lubrication, Lanzhou Institute of Chemical Physics, Chinese Academy of Sciences, \\ 730000 Lanzhou, P. R. China
}

Received 23 February 2013; accepted in revised form 19 April 2013

\begin{abstract}
In this paper, a new method to the preparation of low-dielectric nanoporous polyimide (PI) films was addressed, based on the self-assembly structures of PS-b-P4VP/poly(amic acid) (PAA, precursor of PI) blends. It is found the microphase-separation structure of PS-b-P4VP/PAA is a precondition of the formation of nanoporous structures, which could be achieved by solvent annealing. Nanoporous PI films with spherical pore size of $\sim 11 \mathrm{~nm}$ were obtained by thermal imidization followed by the removal of the PS-b-P4VP block copolymer. The porosity of the nanoporous PI films could be controlled by the weight fraction of the PS-b-P4VP block copolymer. The dielectric properties of the nanoporous PI films were studied, and it was found that the introduction of nanopores could effectively reduce the dielectric constant from 3.60 of dense PI films to 2.41 of nanoporous PI films with a porosity of $26 \%$, making it promising in microelectronic devices. The fabrication method described here could be extended to other polymer systems.
\end{abstract}

Keywords: nanomaterials, block copolymer, dielectric constant, nanoporous polyimide, thin films

\section{Introduction}

With the development of microelectronic devices becoming smaller and lighter, new low dielectric constant materials are needed to replace the current wire insulator of silicon dioxide, thereby reducing the signal delays and electrical power loss in the new generations of large scale integrated circuits [1-3]. Nanoporous polyimide (PI) material, combining the high performance of PI and the lowest dielectric constant of air void $(k \approx 1)$, is thought to be the most promising candidate for use as a next-generation of interlayer dielectrics [4]. Therefore, considerable attention has been focused on the preparation of low-dielectric nanoporous PI films. Besides normal template like poly(ethylene oxide)-polyhedral oligosilsesquioxane (PEO-POSS) nanoparticles [5], silicon nanowires [6], poly[styreneco-(4vinylpyridine)] (PSVP) nanospheres [7], were used to prepare nanoporous PI films, Hedrick and his coworkers have opened a route to the fabrication of nanoporous PI films. The approach they established involves the preparation of block copolymers consisting of a thermally labile block and highly temperature-stable PI block. The block copolymer could be self-assembled into nanoscale domains with labile block as dispersed phase and PI block as matrix. Pore formation can be accomplished by thermolysis of the labile blocks, which leaves pores of sizes and shapes that correspond to those present in the initial copolymer's morphology $[8,9]$. Thanks to their contribution and several other works on the preparation of nanoporous PI films with low dielectric constant based on various PI-based copolymer has been reported [10-13]. However, the synthetic procedures and processing are relatively complicated, furthermore, thermal degradation of the labile com-

\footnotetext{
${ }^{*}$ Corresponding author, e-mail: wangqh@licp.cas.cn

(C) BME-PT
} 
ponent reduces the molecular weight and certain critical mechanical properties of the resulting nanoporous films.

In this study, we described a new method to the preparation of low-dielectric nanoporous PI films using block copolymer as template. Based on the self-assembly structures of block copolymer and poly(amic acid) (PAA, precursor of PI) blends, nanoporous PI films could be obtained after the removal of the block copolymer. Compared to the method Hedrick reported, complex synthetic procedures are avoidable, and the mechanical properties of resultant nanoporous PI films are little influenced because the nanopores come from the simple removal of block copolymer. In this way, the porosity of resultant nanoporous PI films could be controlled by changing of the weight content of block copolymer, thus adjusting the dielectric constant of the films

\section{Experimental procedures \\ 2.1. Materials}

PS-b-P4VP block copolymer $\left(M_{\mathrm{n}}\right.$ PS:P4VP= $11800: 15000 \mathrm{~g} / \mathrm{mol}$ and polydispersity of 1.04 ) was purchased from Polymer Source Inc., Canada, and used as received. 3,3',4,4'-biphenyltetracarboxylic dianhydride (BPDA), 4,4'-oxydianiline (ODA), and N-Methy1-2-pyrrolidone (NMP), benzene were analytical chemical reagents and purchased from Tianjin Chemical Reagents Company, Tianjin, China.

\subsection{Synthesis of poly(amic acid) homopolymer}

PAA homopolymer was prepared by solution polymerization in NMP. Into a reaction flask fitted with a mechanical stirrer and Ar inlet were charged $1.8 \mathrm{~g}$ of ODA and $30 \mathrm{~g}$ of NMP. Stirring was begun, and after the ODA has dissolved, $2.698 \mathrm{~g}$ of BPDA powder was added gradually to the stirring solution of the diamine over $40 \mathrm{~min}$. The reaction mixture was reacted at $0^{\circ} \mathrm{C}$ for $24 \mathrm{~h}$ in $\mathrm{Ar}$ atmosphere to yield a viscous PAA solution.

\subsection{Preparation of nanoporous polyimide thin films}

The procedure used to fabricate nanoporous PI films is summarized in Figure 1. The block copolymer PS-b-P4VP and PAA homopolymer was first dissolved in NMP, to make polymer solution with a concentration of $5 \mathrm{wt} \%$. The NMP solution contain- ing $5 \mathrm{wt} \%$ polymer mixtures was stirred for more than $24 \mathrm{~h}$ at room temperature. PS-b-P4VP/PAA blends in films with a thickness of $\sim 100 \mathrm{~nm}$, were prepared on a silicon wafer by spin-coating at $2000 \mathrm{rpm}$ for $4 \mathrm{~min}$, followed by drying under vacuum at room temperature to remove the residual NMP. The as-spun PS-b-P4VP/PAA films were solvent annealed in a saturated mixture vapor of benzene and NMP at $80^{\circ} \mathrm{C}$ to achieve self-assembly microstructures. The self-assembled PS-b-P4VP/ PAA films were thermally treated to convert PAA to PI at an elevated temperature: $120^{\circ} \mathrm{C}(1 \mathrm{~h}), 180^{\circ} \mathrm{C}$ $(1 \mathrm{~h}), 250^{\circ} \mathrm{C}(1 \mathrm{~h})$, and $300^{\circ} \mathrm{C}(1 \mathrm{~h})$. Then, nanoporous PI films with a thickness of $\sim 100 \mathrm{~nm}$, were obtained after the removal of PS-b-P4VP block copolymer by solvent extraction in dichloromethane for $24 \mathrm{~h}$ at $50^{\circ} \mathrm{C}$.

\subsection{Characterization}

AFM studies were operated at a Nanoscope IIIa multimode atomic force microscope (Digital Instruments, VEECO, USA) at the tapping mode. Transmission electron microscope (JEM-2010, JEOL Ltd., Japan) operating at an accelerating voltage of $200 \mathrm{kV}$ was used to examine the morphology of PSb-P4VP/PAA and nanoporous PI films. Films for TEM were prepared on silicon substrates having a thick layer of silicon oxide. These films were floated onto the surface of a $5 \mathrm{wt} \%$ hydrofluoric acid solution, transferred to a water bath, and then picked up by a $\mathrm{Cu}$ grid. To clearly observe the interior structures of PS-b-P4VP/PAA, iodine was employed as staining agent to selectively contrast PAA chains and P4VP blocks. The samples were placed in vapors of iodine crystals for $2 \mathrm{~h}$ at $35^{\circ} \mathrm{C}$. The porosity $(V)$ of the nanoporous PI thin films was obtained by calculating from refractive index according to the Maxwell-Garnett modeling as shown by Equation (1) [14]:

$$
V=1-\left[\frac{\left(n_{\mathrm{p}}^{2}-1\right)\left(n_{\mathrm{s}}^{2}+2\right)}{\left(n_{\mathrm{p}}^{2}+2\right)\left(n_{\mathrm{s}}^{2}-1\right)}\right]
$$

where $n_{\mathrm{p}}$ and $n_{\mathrm{s}}$ is refractive index of nanoporous and dense PI thin films, respectively. The dielectric constant of the nanoporous PI films was determined using the Maxwell equation, Equation (2) [15]:

$\varepsilon \cong 1.1 \cdot n^{2}$

where $n$ is the refractive index. The refractive index was measured with a variable-angle multi-wave- 


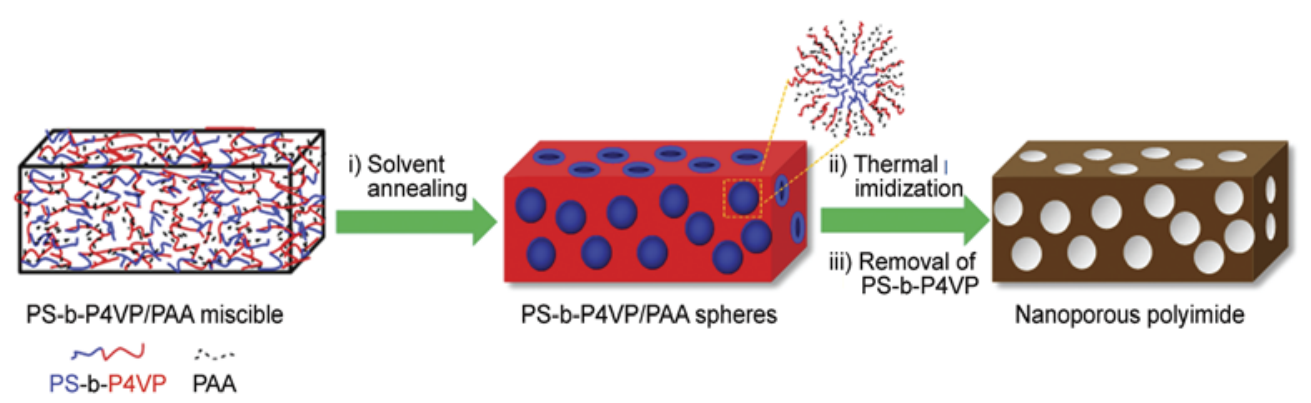

Figure 1. Schematic figure of fabrication of nanoporous PI films

length ellipsometer (L116E, Gaertner Ltd., USA) with wavelengths at $632.8 \mathrm{~nm}$.

\section{Results and discussion}

Prior to discussion, it should be noted that there exists a strong interpolymer hydrogen-bonding interaction between PAA and P4VP blocks, leading to the formation of P4VP/PAA complex (Figure 2). The selective adsorption of PAA on P4VP blocks could increase the interaction parameter difference between PS and P4VP/PAA phases, resulting in the strong segregated microphase separation [16]. TEM was performed to explore the interior structure of the PS-b-P4VP/PAA thin film. After $I_{2}$ staining, the PAA and P4VP microdomains are dark, and the PS components appear light. For the directly spincoated thin film, it is noticed that Figure 3a represents a homogenous phase. During the formation of PS-b-P4VP/PAA films by spin-coating from NMP solution, not any microphase-separation structures could be obtained due to non-selectivity of NMP and high solvent evaporation rate [17]. We have attempted to prepare porous PI films from the directly spin-coated PS-b-P4VP/PAA film after conversion of PAA into PI and removal of PS-b-
P4VP, but it failed. The results demonstrate that the miscible phase of the as-spun PS-b-P4VP/PAA film could not produce any porous structures. The PS-bP4VP/PAA blends thin film was solvent-annealed in benzene/NMP vapor mixture to achieve microphase-separation [17]. As shown in Figure 3b, it represents a microphase-separation structure consisted of PS spherical domains dispersed within P4VP/PAA matrix.

Figures 4a-c show the AFM images of self-assembled PS-b-P4VP/PAA films with different PAA wt $\%$. It is found spherical microphase-separation structures as well as few of wire-like structures formed. Although PAA weight content in the three PS-bP4VP/PAA films is different, the diameter of spherical microphase-separation structures, $\sim 19 \mathrm{~nm}$, is nearly the same. The self-assembled PS-b-P4VP/ PAA films were thermally treated to convert PAA to PI. FIIR results as shown in Figure 5 confirmed the successful imidization of PAA. Figures $4 d-4 f$ describe the AFM images of the resultant PS-bP4VP/PI films after thermal imidization. Compared to PS-b-P4VP/PAA films, wire-like structures disappear and size of spherical structures increased to $\sim 31 \mathrm{~nm}$. During the conversion of PAA to PI by

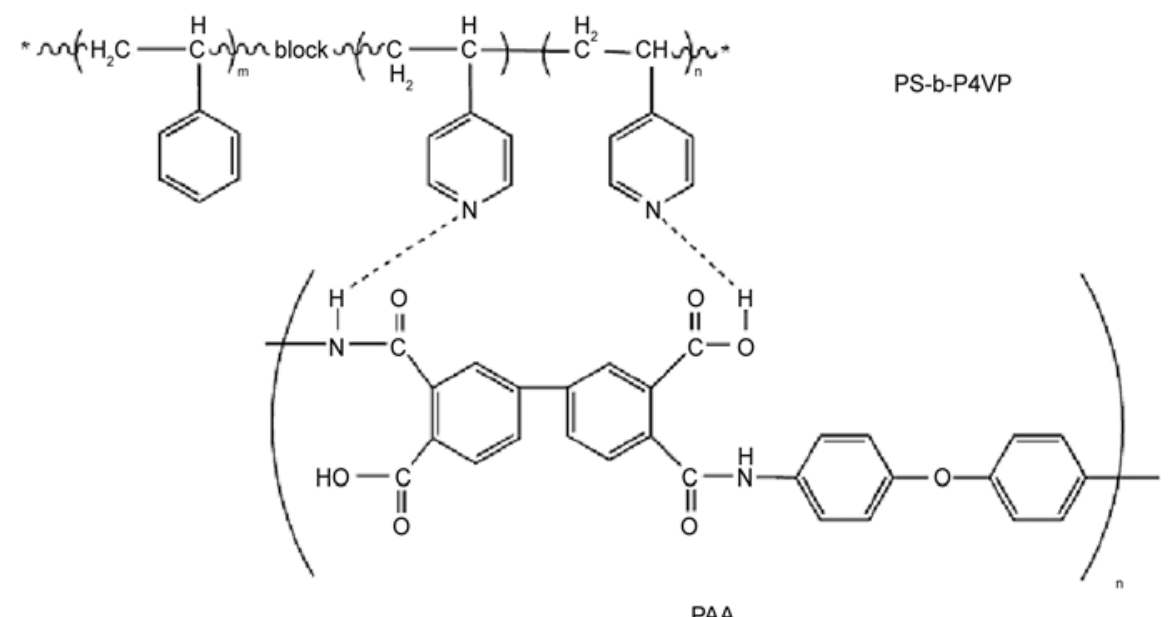

Figure 2. Schematic representation of possible interactions between PAA and PS-b-P4VP block copolymer 


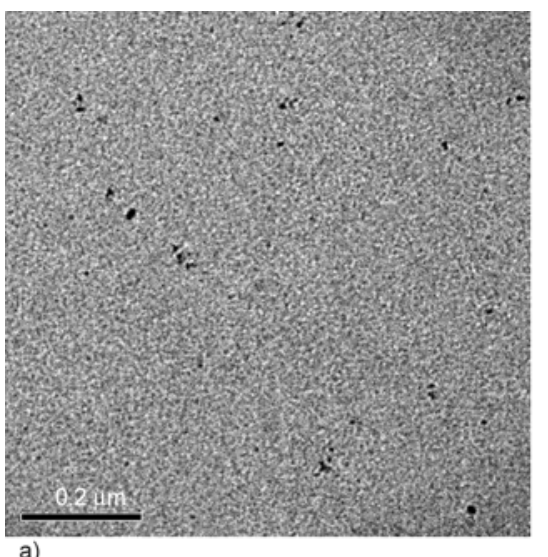

a)

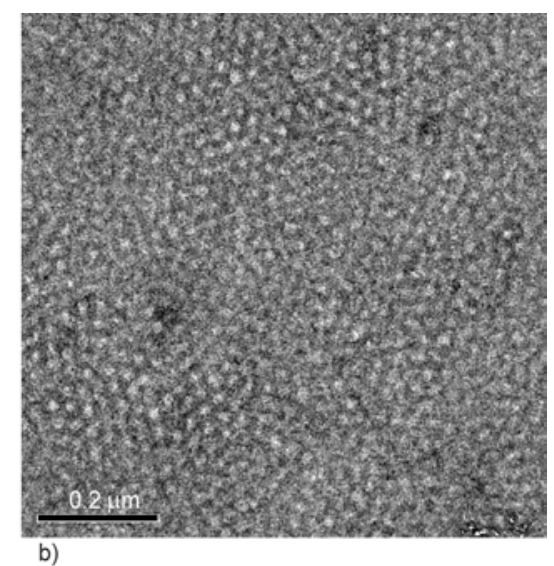

b)

Figure 3. TEM images of PS-b-P4VP/PAA blends thin film with PAA wt $\%$ of 50\% (a) before and (b) after solvent annealing in benzene/NMP mixture vapor

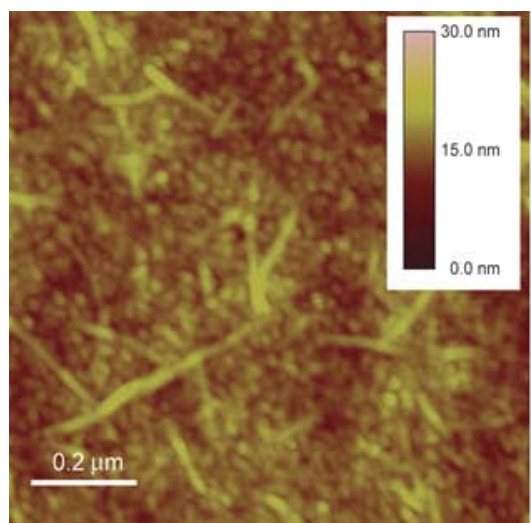

a)

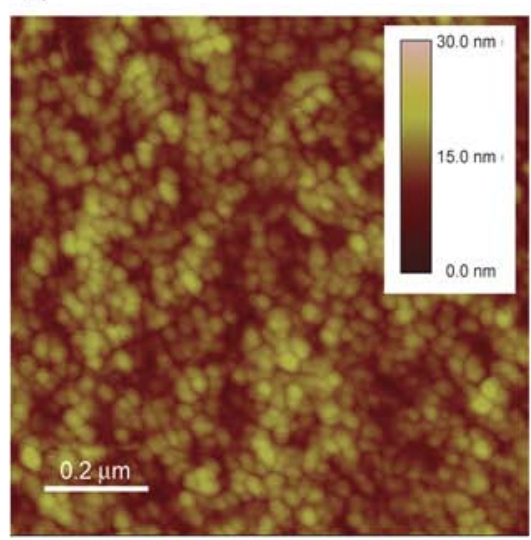

d)

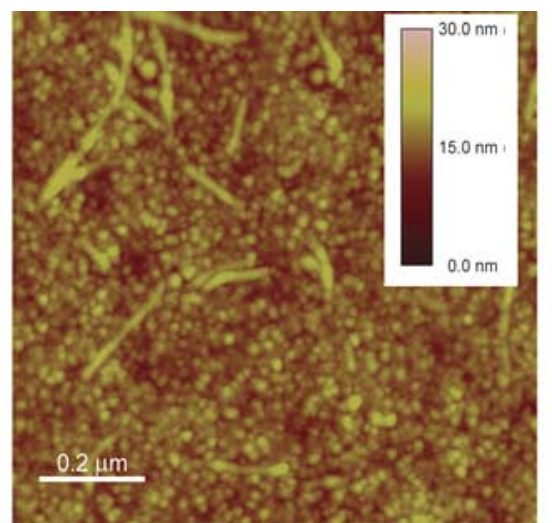

b)

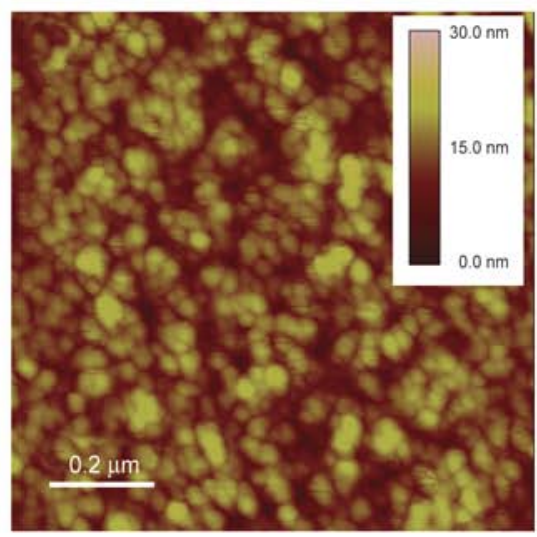

e)
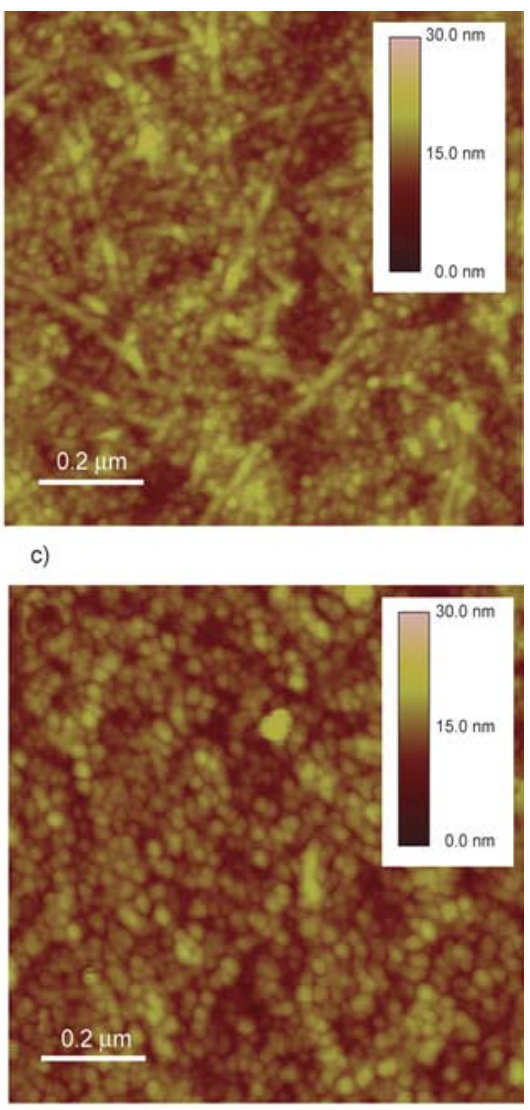

f)

Figure 4. AFM images of PS-b-P4VP/PAA self-assembled films with different PS-b-P4VP wt $\%$ of (a) $40 \%$, (b) $50 \%$ and (c) $60 \%$. (d), (e) and (f) are AFM images of resultant PS-b-P4VP/PI films after thermal imidization

thermal annealing, PI chains were separated from P4VP blocks because the inter-polymer hydrogenbonding interaction between PAA and P4VP blocks was destroyed, thus leading to the increase of sphere size and disappearance of wire-like structures.

Nanoporous PI films could be obtained from PS-bP4VP/PI films after removal of PS-b-P4VP block copolymer by solvent extraction in dichloro- methane. Here, solvent extraction of PS-b-P4VP was used to replace traditional thermal decomposition to prevent the collapse of the porous structure during thermolysis at high temperature. As shown in Figure 5, disappearance of the peak at $697 \mathrm{~cm}^{-1}$, corresponding to carbon-nitrogen distortion vibration of the pyridine ring, revealed the complete removal of PS-b-P4VP block copolymer. Figure 6 demonstrates the TEM images of the resultant PI 


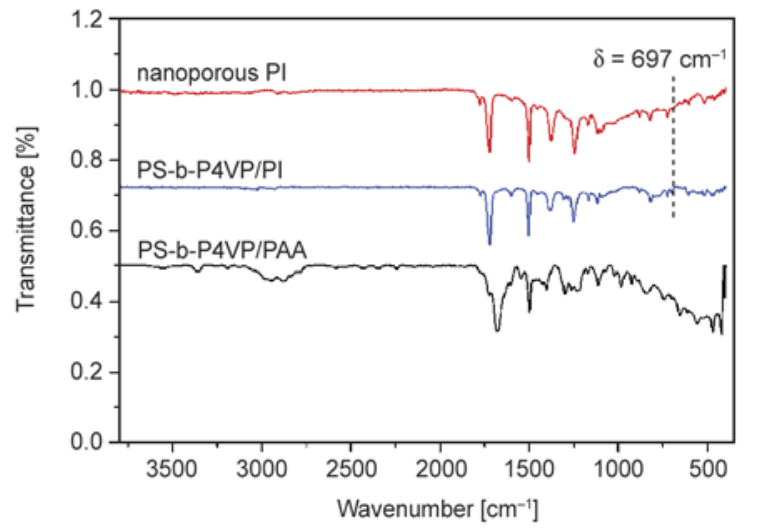

Figure 5. FT-IR spectrums of PS-b-P4VP/PAA, PS-b$\mathrm{P} 4 \mathrm{VP} / \mathrm{PI}$ and nanoporous PI thin films

films. The nanoscale pores of the films are clearly evident with the white areas. Low contrast in the TEM image could be attributed to the overlapping of projections of nanopores in the thickness direction. Nanoporous structures with nearly the same diameter of $\sim 11 \mathrm{~nm}$ were found, formed in three kinds of PI films. It reveals that the weight content of PS-b-P4VP block copolymer did not have any obvious influence on the pore size of resultant nanoporous PI films. It should be noted, in our study here, only three kinds of PS-b-P4VP/PAA films and as-prepared nanoporous PI films were discussed, because the other PS-b-P4VP/PAA films with PS-b-P4VP wt $\%$ not in the range of $40 \sim 60 \%$ could not form proper microphase-separated structures to fabricate nanoporous PI films.

The closed and nanoscale pores of the prepared PI films allow potential applications in microelectronic devices. Table 1 demonstrates the porosity and dielectric constant of the nanoporous PI films. It can be seen the porosity of the films could be adjusted by PS-b-P4VP weight content in PS-b-
Table 1. Refractive index, porosity and dielectric constant of nanoporous PI films prepared from PS-bP4VP/PAA blends films with different PS-bP4VP wt $\%$

\begin{tabular}{|l|c|c|c|}
\hline \multicolumn{1}{|c|}{ Sample } & $\begin{array}{c}\text { Refractive } \\
\text { index }\end{array}$ & Porosity $^{\text {a }}$ & $\begin{array}{c}\text { Dielectric } \\
\text { constant }\end{array}$ \\
\hline $\mathrm{PI}$ & 1.81 & - & 3.60 \\
\hline $\mathrm{PI}_{40 \%}$ & 1.60 & 0.20 & 2.82 \\
\hline $\mathrm{PI}_{50 \%}$ & 1.55 & 0.26 & 2.64 \\
\hline $\mathrm{PI}_{60 \%}$ & 1.48 & 0.34 & 2.41 \\
\hline
\end{tabular}

${ }^{a}$ Determined according to Maxwell-Garnett modeling [14]

P4VP/PAA blends films. As the result shows, the dielectric constant of the films decreases with the increasing porosity. The dielectric constant varies from 2.82 to 2.41 for the nanoporous PI films, significantly lower than that of the dense PI film (3.60). It indicates that the incorporation of nanopores successfully reduces the dielectric constant of PI films.

\section{Conclusions}

Nanoporous PI films with low dielectric constant were prepared, based on the microphase-separated structures of PS-b-P4VP/PAA. The incorporation of nanopores could effectively reduce the dielectric constant of the resultant PI films. Porosity of the nanoporous PI films could be controlled by the weight content of PS-b-P4VP block copolymer, thereby adjusting the dielectric constant of the films. The low dielectric constant of the prepared nanoporous PI films make it promising in the microelectronics.

Since block copolymer have the ability to selfassembly into versatile nanoscale morphologies, it is expected multifarious nanoporous structures could be prepared and the effect of these structures on the dielectric property of the nanoporous PI films could

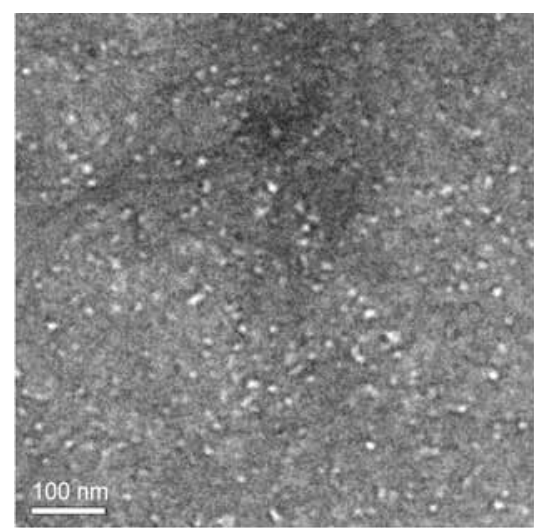

a)

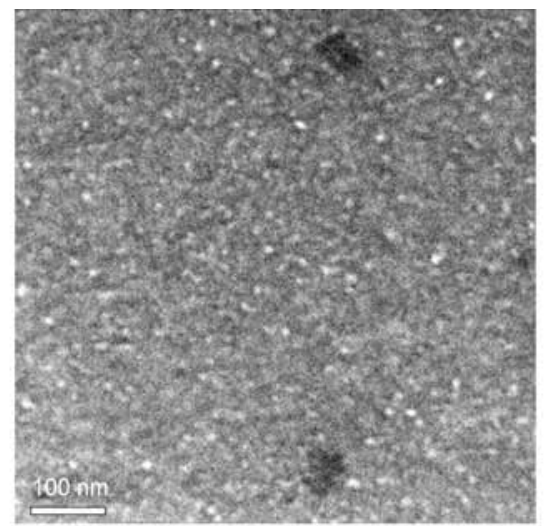

b)

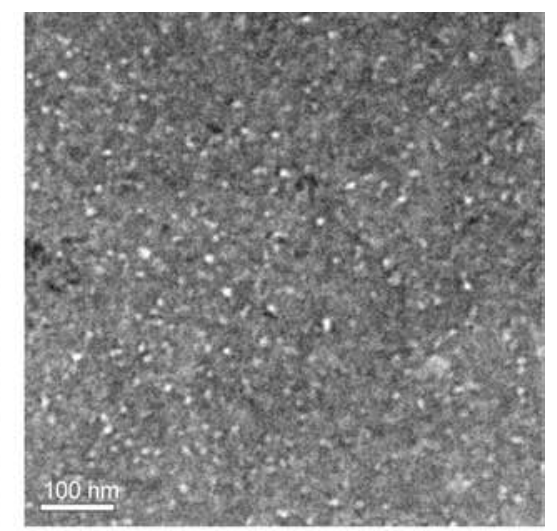

c)

Figure 6. TEM images of nanoporous PI films obtained from PS-b-P4VP/PAA self-assembled films with different PS-bP4VP wt $\%$ of (a) $40 \%$, (b) $50 \%$ and (c) $60 \%$ after removal of PS-b-P4VP 
be studied in the future work. The method described here is simple and controllable, which could be applied to the other polymer systems.

\section{Acknowledgements}

The authors would like to acknowledge the financial support of the National Science Foundation for Distinguished Young Scholars of China (Grant No.51025517) and the National Defense Basic Scientific Research Project (A1320110011).

\section{References}

[1] Homma T.: Low dielectric constant materials and methods for interlayer dielectric films in ultralargescale integrated circuit multilevel interconnections. Materials Science and Engineering R: Reports, 23, 243-285 (1998).

DOI: 10.1016/S0927-796X(98)00012-6

[2] Geng Z., Lu Y., Zhang S., Jiang X., Huo P., Luan J., Wang G.: Synthesis and characterization of novel adamantane-based copoly(aryl ether ketone)s with low dielectric constants. Polymer International, in press (2013).

DOI: $10.1002 /$ pi.4515

[3] Cho E-B., Mandal M., Jaroniec M.: Periodic mesoporous benzene-silicas prepared using boric acid as catalyst. Chemistry of Materials, 23, 1971-1976 (2011). DOI: $10.1021 / \mathrm{cm} 200166 \mathrm{f}$

[4] Zhao G., Ishizaka T., Kasai H., Oikawa H., Nakanishi H.: Fabrication of unique porous polyimide nanoparticles using a reprecipitation method. Chemistry of Materials, 19, 1901-1905 (2007).

DOI: $10.1021 / \mathrm{cm} 062709 \mathrm{w}$

[5] Lee Y-J., Huang J-M., Kuo S-W., Chang F-C.: Lowdielectric, nanoporous polyimide films prepared from PEO-POSS nanoparticles. Polymer, 46, 10056-10065 (2005).

DOI: $10.1016 /$ j.polymer.2005.08.047

[6] Kim W., Lee M-K.: Fabrication of a porous polyimide membrane using a silicon nanowire array as a template. Materials Letters, 63, 933-936 (2009). DOI: $10.1016 /$ j.matlet.2009.01.060

[7] Xu Z-K., Liu Z-M., Xiao L., Dai Q-W., Kou R-Q.: Polyimide/poly(styrene-co-4-vinylpyridine) nanocomposite films as precursors for the preparation of polyimide nanofoams. Journal of Dispersion Science and Technology, 23, 519-527 (2002).

DOI: $\underline{10.1081 / D I S-120014020}$
[8] Olson D. A., Chen L., Hillmyer M. A.: Templating nanoporous polymers with ordered block copolymers. Chemitry of Materials, 20, 869-890 (2008). DOI: $10.1021 / \mathrm{cm} 702239 \mathrm{k}$

[9] Wang Y., Li F.: An emerging pore-making strategy: Confined swelling-induced pore generation in block copolymer materials. Advanced Materials, 23, 21342148 (2011).

DOI: $10.1002 / \mathrm{adma} .201004022$

[10] Hedrick J. L., Miller R. D., Hawker C. J., Carter K. R., Volksen W., Yoon D. Y., Trollsås M.: Templating nanoporosity in thin-film dielectric insulators. Advanced Materials, 10, 1049-1053 (1998).

DOI: 10.1002/(SICI)1521-4095(199809)10:13<1049:: AID-ADMA1049>3.0.CO;2-F

[11] Hedrick J. L., Russell T. P., Labadie J. W., Lucas M., Swanson S. A.: High temperature nanofoams derived from rigid and semi-rigid polyimides. Polymer, 36, 2685-2697 (1995).

DOI: 10.1016/0032-3861(95)93645-3

[12] Hedrick J. L., Russell T. P., Sanchez M., DiPietro R., Swanson S.: Polyimide nanofoams from caprolactonebased copolymers. Macromolecules, 29, 3642-3646 (1996).

DOI: $10.1021 / \mathrm{ma950903q}$

[13] Hedrick J. L., Carter K. R., Labadie J., Miller R. D., Volksen W., Hawker C. J., Yoon D. Y., Russell T. P., McGrath J. E., Briber R. M.: Nanoporous polyimides. in 'Advances in polymer science: Progress in polyimide chemistry’ (ed.: H. R. Kricheldorf), SpringerVerlag, Berlin, Vol 141, 1-43 (1999).

DOI: $10.1007 / 3-540-49814-11$

[14] Nitta S. V., Pisupatti V., Jain A., Wayner P. C., Gill W. N., Plawsky J. L.: Surface modified spin-on xerogel films as interlayer dielectrics. Journal of Vacuum Science and Technology B, 17, 205-212 (1999).

DOI: $10.1116 / 1.590541$

[15] Matsumoto T.: Nonaromatic polyimides derived from cycloaliphatic monomers. Macromolecules, 32, 49334939 (1999).

DOI: $10.1021 / \mathrm{ma} 9903862$

[16] Wang C., Wang T., Pei X., Wang Q.: Shell-corecorona aggregates formed from poly(styrene)-poly(4vinylpyridine) block copolymer induced by added homopolymer via interpolymer hydrogen-bonding. Polymer, 50, 5268-5275 (2009).

DOI: $10.1016 /$ j.polymer.2009.09.028

[17] Wang C., Wang T., Wang Q.: Solvent annealing assisted self-assembly of hydrogen-bonded interpolymer complexes of AB block copolymer/C homopolymer in thin film. Polymer, 51, 4836-4842 (2010). DOI: $10.1016 /$ j.polymer.2010.08.047 